\title{
LE RAVIN SAUREAULT ET SA CORRECTION
}

\author{
par Roch Delisle, B.A., I.F.
}

$A$ L'EXTREMITE nord de la paroisse de St-Clet s'élève, de la plaine argileuse couvrant le comté de Soulanges et une bonne partie du conté de Vaudreuil, un plateau sableux dont l'altitude moyenne domine de quelque $100^{\prime}$ celle de la plaine qui le circonscrit sur les deux tiers de son pourtour.

Lorsque se forma dans le passé le réseau de drainage des eaux de ce plateau vers les basses terres, il y eut, en raison de la dénivellation existante et du caractère meuble des terrains, un affouillement prononcé de la partie du plateau limitrophe de la plaine. Ceci donna naissance dans la partie marginale de la terrasse à un nombre important de ravins ou coulées de largent et de profondeur variées et s'avançant plus ou moins profondément dans l'intérieur du plateau.

Puis à la période de ravinement intense concordant avec la formation du système hydrographique du platean, suivit sans doute, avec l'apparition de la forêt sur ce plateau, une période durant laquelle l'action érosive des eaux fut moindre. Ceci permit à la forêt de s'établir sur les versants et dans le fond des ravins et d'empêcher dès lors l'eau de ruissellement et de fonte des neiges de modifier sensiblement la forme de ces derniers.

Parmi les coulées ainsi formées, il y avait celle qui fut plus tard dénommée coulée Saureault. Sa longucur était de $3,417^{\prime}$ avec une dénivellation probable de $86^{\prime}$ donnant une pente moyenne de $2.5 \%$. Elle ne servait que pour l'écoulement passager des eaux de fonte des neiges et ne donnait naissance par des sources à un ruisseau qu'à peu de distance de la plaine.

L'équilibre maintenu par la forêt entre la force vive des caux et la résistance du sol fut rompu cependant par les déboisements que firent les colons sur le plateau et un nouvel affouillage des terrains recommença vers 1909 pour s'amplifier par la suite. De ce fait, de nouveaux ravins se creusèrent. Comme presque tout le bassin de réception des eaux s'écoulant par la coulée primitive Saureault fut déboisé ainsi que la coulée elle-même à sa partie supérieure, il se fit un ravinement dans la coulée même que l'on désigna sous le nom de ravin Saureault.

Après l'achat des terrains nécessaires par les municipalités de St-Clet et de St-Lazare, le Service forestier de Québec, répondant à l'appel de ces municipalités, entreprit la correction de ces ravins et l'extinction des ravines qui les avaient formés et qui continuaient à les agrandir. Les premiers travaux débu- 
tèrent en 1930 et furent couronnés de succès. La correction du ravin Saureault, le dernier à être corrigé, fut entreprise en fin d'octobre 1934 et à peu près terminée le ler juillet 1935.

Le ravin Saureault était une tranchée, longue de $1560^{\prime}$, taillée dans des terrains $100 \%$ sableux, à peu près dénudée de toute végétation sur 625' de sa tête vers l'aval, partiellement boisée sur le reste de sa longueur, d'une profondeur variant entre 20 et $35^{\prime}$ et d'une largeur allant de 30 à $100^{\prime}$. Sur son flanc ouest, quatre commencements de ravins latéraux appelés "têtes secondaires" dont la plus importante d'une longueur de 175'. Dans son fond, un ruisseau qui originant à quelque $60^{\prime}$ en aval de la tête de cette tranchée ne tarde pas à avoir un déhit de plusieurs gallons à la minute, eau fournie par une infinité de sources. Dernier détail: le fond du ravin à la tête coupée d'apic de celui-ci était en contre-bas de $12^{\prime}$ du fond de la coulée primitive dans laquelle comme dit plus haut le ravin se forma.

Le ravin Saureault, par ses dimensions, par la grosseur du ruisseau coulant dans son fond, par le coup d'eau de fonte des neiges qu'il recevait au printemps, par sa position même dans une coulée, était le ravin le plus dangereux, celui dont le creusage avait donné et continuait de donner le plus de sabłes à la plaine. Il était aussi le ravin le plus difficile de correction de tous les ravins travaillés.

Trois facteurs concouraient à son agrandissement et étaient responsables d'une façon directe ou indirecte de la descente des sables dans la plaine: $1^{\circ}$ Lo soleil $2^{\circ}$ Le vent $3^{\circ}$ L'eau. Ce dernier élément peut se diviser en trois: l'eau de pluie, l"eau de source constituant en temps normal le régime normal du ruisseau et l'eau de fonte des neiges.

Comme sus-dit, une bonne partie des flancs du ravin étaient dépourvus de toute végétation. C'est donc dire qu'au moindre soleil, le sable de ces talus à pente raide s'échauffait rapidement et perdait toute cohérence. Il glissait alors sous l'action de la pesanteur au fond du ravin pour être entraîné par les filets d'eau.

Les vents fréquents, qui, de la plaine, soufflent sur le plateau, en arrachant de leur position les particules sableuses lorsqu'asséchées, rongeaient la partie supérieure des talus. Sous leur action érosive, cet endroit ne tardait pas à avoir un profil vertical. D'où éboulis, glissements de sable au fond du ravin, puis nouvelle attaque par les vents. Ce sable éboulé ainsi que les particules qui soulevées par le vent retombaient dans le ruisseau étaient entraînés aussitôt par les eaux.

Les eaux sauvages, résultat d'orages violents, de pluies torrentielles, en dévalant en vitesse les flancs du ravin affouillaient également et remettaient au ruisseau les sables qu'elles avaient réussi à entraîner au fond de la tranchée.

Le ruisseau coulant dans le ravin accomplissait un travail d'érosion encore 
plus prononcé que celui fait par le soleil, les vertts et les eaux de ruissellement. En rongeant constamment le pied des berges et en le rendant instable, it provoquait sur son parcours de nouveaux éboulis par lesquels le ravin allait tojours en s'élargissant. Ses eaux transportaient, roulaient sans cesse des sables vers la plaine.

Au printemps, durant quelque 24 heures, le long couloir que constitue la partie de la coulée primitive Saureault non ravinée et qui fait communiquer les eaux de fonte des neiges d'un bassin de réception d'un mille carré approximativement avec le ravin, déverse dans celui-ci $2^{\prime}$ d'épais d'eau sur une largeur de $4^{\prime}$ environ. C'est le coup d'eau si redouté. Ce torrent faisait avancer la tête de la tranchée chaque année. De plus, dans sa course vers la plaine, il attaquait sérieusement les berges, principalement dans le fond des courbes, affouillait le fond du ravin et reprenait les matières que le ruisseau en temps normal avait dû déposer à la sortie du ravin pour aller répandre le tout plus loin sur les terres cultivées. D'où, dégâts causés par le recouvrement des terres fortes de la plaine par une épaisse couche de sable délavé tout-à-fait improductif. D'autres dommages non moins graves résultaient du fait que le ruisseau dans la plaine se trouvant rempli par les sables ne pouvait plus égoutter les terres et cela à une période de l'année où ces terres avaient le plus besoin d'être drainées.

Pour arrêter les sables de descendre dans la plaine, ce qui revient à dire pour éteindre la ravine constituée par le coup d'eau de fonte des neiges et pour corriger le ravin, il fallait: $1^{\circ}$ Réinstaller la forêt dans la partie non ravinée de la coulée Saureault et sur ses bords.

$2^{c}$ Travailler les têtes du ravin de façon à ce que les eaux de fonte des neiges ou celles de ruissellement ne pussent point les affouiller davantage et les faire avancer de nouveau.

$3^{\circ}$ Stabiliser le fond du ravin et le pied de ses berges afin d'empêcher le ravin de se creuser davantage et les berges de subir de nouveaux éboulis par suite de l'érosion par l'eau de leur pied.

$4^{c}$ Mettre la partie supérieure des talus à l'abri de l'action érosive du vent afin que ces talus pussent conserver leur angle de repos tel que donné par le terrassement lors des travaux de correction.

$5^{\circ}$ Empêcher les talus d'être ravinés par les eaux sauvages et soustraire leurs sables à l'action dessicative du soleil.

Tous les découverts à la tête du ravin et sur ses bords furent reboisés d'épinette et de pin blanc avec un espacement des plants entre eux de $3^{\prime}$, afin d'obtenir un recouvrement aussi rapide que possible du sol. Le jour où la forêt sera assez haute pour exercer une influence sur la vitesse de fonte des neiges, la ravine ira en diminuant pour s'éteindre un jour. 
Les têtes s'érodaient parce que, taillées d'apic, l'eau en coulant mangeait leur base. Faute d'appui, le sol s'affaissait sous son propre poids au fond de la tranchée pour être ensuite entrâné par l'eauu. Puis, un nouveau sapement recommençait.

Toutes les têtes furent comblées de pierre afin de protéger leur base et régulariser la pente d'écoulement. La cavité emplie, on prolongea le revêtement de pierre jusqu'au fond du ravin obligeant ainsi les eaux qui temporairement arrivent à ces têtes de couler tout le long du talus sur un lit pierreux compact.

A la tête principale du ravin cependant, le remblai de pierre reçut une forme telle que l'eau doit continuer de tomber en chûte. Ceci pour casser' la vitesse acquise du coup d'eau de fonte des neiges à son arrivée au ravin. En même temps, ceci permit de corriger cette tête plus économiquement.

Les filets d'eau à leur arrivée à la tranchée tombent présentement sur un lit de pierres de plusieurs pieds d'épaisseur qui préserve ainsi la base de la tête de toute érosion. Comme ce lit est loin d'avoir une surface unie, les filets d'eau rencontrent des obstacles sur lesquels ils perdent une partie de leur vitesse avant de continuer leur route.

Les têtes, telles que corrigées, sont maintenant des couloirs permettant aux eaux de descendre au ravin sans affouiller. Un remblai de plaques de gason courant à quelques pieds de la crête des talus du ravin s'efforce d'amener à ces couloirs les eaux sauvages et celles de fonte des neiges.

Lc fond du ravin et le pied de ses herges furent stabilisés avec de la pierre également.

Après rectification des courhes dangereuses du ruisseau au fond du ravin et après avoir donné à ce fond une section aussi grande que possible afin de diminuer l'épasseur de la lame d'eau en l'étendant, il fut posé de part et d'autrc du fond du ravin un mur de pierre dont la mission est de préserver le pied des berges de l'action érosive de l'eau et de servir d'appui au sable descendu du sommet des talus lors du terrassement de ceux-ci. Ce mur fut fait en grande partic de houlders cassés ì la dynamite afin d'avoir des pierres aux fáces assez régulières car, il va sans dire, aucun nortier ne fut employé. La hauteur du mur dépasse partout le niveau maximum des eaux en temps de plus grande crue. A certains endroits, on augmental sa hauteur pour pouvoir abaisser davantage les pentes des talus.

Le mur terminé, le fond du ravin reçut un revêtement de cailloux, de pierres cassées afin d'empêcher l'eau de le creuser davantage et afin de prévenir que les murs latéraux ne soient sapés à leur base. Dans tous les interstices des pierres, il fut inséré des bouts de saules qui en prenant racine travailleront égalemerst à la stabilisation du sol. 
Cet empierrement du fond du ravin donna des résultats immédiats. L'eau, qui, auparavant n'était point potable du fait qu'elle était toujours chargée de particules sableuses que dans son mouvement d'érosion elle transportait, est aujourd'hui des plus claires. De plus, sa vitesse d'écoulement et par suite sa force érosive est beaucoup diminuée du fait qu'à tous les moments les filets d'eau rencontrent une roche sur laquelle ils perdent une partie de leur vitesse acquise. La stabilisation du fond du ravin a aussi permis d'avoir à la place de terrains constamment en mouvement quelque chose de stable sur lequel la végétation peut s'établir. Et de fait, en plusieurs endroits, l'on peut déjà voir une belle venue d'herbes aquatiques.

La correction des talus, une fois leur pied stabilisé, fut faite de la façon suivante:

Par un terrassement approprié, on fit d'abord disparaître de ces talus tous les endroits où le profit de la pente était trop raide et l'on donna partout un angle de déclivité permettant un repos certain du terrain. La jonction de la ligne de pente des flancs du ravin avec le rebord de celui-ci se fit suivant une ligne courbe.

Le terrassement fait, on érigea sur les talus des barrages enfouis de saule. Ces barrages disposés perpendiculairement à la pente sont faits de la façon suivante: après avoir pratiqué une tranchée d'environ 8" de profondeur par autant de largeur, des piquets étaient enfoncés au milieu de cette tranchée et les branches de saules de pas plus de d'1" de diamètre tressées sur ces piquets, jusqu’à ce que le harrage atteignit le niveau du sol. Puis la tranchée était remplie et la terre soigneusement foulée le long et sur les barragres.

Le nombre de barrages et leur distance entre eux varièrent suivant les conditions de stabilité de chaque endroit.

La mise en place du saule faite, on recouvrit les talus sur une largeur d'une quinzaine de pieds à partir du mur d'appui vers leur sommet de branches d'arbres, d'arbres entiers et de tous les déchets qui se trouvaient près de là. Tous ces bois serviront à conserver au sable sa fraîcheur tout en l'empêchant de glisser par-dessus le mur d'appui.

Au-dessus de la zone des branches, les talus furent recouverts jusqu'au rebord du ravin d'une épaisse couche de paille d'avoine principalement. Avant d'étendre la paille, des mauvaises graines recueillies à la Coopérative de mil et trèfle de St-Clet furent épandues à profusion sur le sable dans l'espérance qu'une partie de ces graines de toutes sortes germeraient et gazonneraient le sable dénudé. Commes ces graines ne coûtaient que le trouble de les mettre en sac, il y eut largesse dans leur emploi.

Sur la couverture de paille furent placées de minces branches d'arbres afin d'alourdir la paille. Enfin, sur le tout, des longerons, d'environ 8 " en 
diamètre et de longueurs variées pour faciliter leur mise en place et leur repos parfait sur le sol, furent disposés transversalement à la pente et à une distance moyenne de $4^{\prime}$ les uns des autres. Retenus en place par des piquets, ces longerons en plus d'empêcher le vent de soulever la paille serviront à fixer le sable, à briser la vitesse d'écoulement le long des pentes. Ils permettront de plus, chose très utile, de descendre et de remonter les talus du ravin sans provoquer d'éboulis de sable. Leur présence sera très utile, en effet, pour toute plantation d'arbres qu'il sera nécessaire de faire sur les flancs du ravin.

La partie supérieure des talus fut recouverte de paille afin d'arracher immédiatement à l'emprise du vent les sables à cet endroit, de conserver l'humidité à ces sables, de les engraisser et de faciliter ainsi l'installation d'une végétation quelconque. Après la pose de la paille, les grains d'avoine et de sarrazin que cette paille contenait levèrent aussitôt avec ici et là des fugères et des chardons. Le sarrazin et l'avoine ne reparaîtront pas ce printemps mais leurs déchets augmentés de ceux de la paille faciliteront l'installation de fraisiers, de framboisiers, de ronces, de vinaigriers, de bouleau, de tremble, etc., qui assureront, avec les pins blancs et les pins de montagne qui seront plantés aux endroits voulant se découvrii, l'établissement d'un tapis végétal qui soustraira complètement les sables à l'action érosive du vent et les rendra plus difficiles d'affouillement.

Une simple plantation de pin blanc et de pin de montagne n'aurait pas suffi à ramener immédiatement un tapis végétal. Entre les plants, le sable serait resté à nu et l'eau et le vent n'auraient pas tardé à déraciner les plants.

Malgré que le coup d'eau fut très fort ce printemps, le ravin Saureault tel que corrigé n'a pas bougé et l'eau a passé sans descendre dans la plaine les sables qu'elle avait coutume d'y apporter. A mesure que le saule et la végétation de toute sorte qui s'installent dans le ravin prendront de l'ampleur, que le reboisement fait à la tête du ravin prendra de l'âge le danger de ravinement diminuera pour disparaître dans un avenir prochain. 